\title{
HA del castillo!! Historia y Acción social en torno a la las fortalezas españolas
}

\section{Angela López Sabatera, Xavier Laumain ${ }^{b}$, Vanesa García López de Andujar ${ }^{c}$ y Juan Eloy Gil García ${ }^{\text {d }}$}

${ }^{a}$ Coordinadora de Didáctica Patrimonial en ARAE Patrimonio y Restauración SLP, C/Llano de la Zaidia 20, 2, 46009 Valencia. araekids@arae.es, ARAE Patrimonio y Restauración SLP, C/Llano de la Zaidia 20, 2, 46009 Valencia. xavier@arae.es,

${ }^{c}$ Departamento de Didáctica Patrimonial en ARAE Patrimonio y Restauración SLP, C/Llano de la Zaidia 20, 2, 46009 Valencia. vagarlo77@gmail.com y ${ }^{\mathrm{d}}$ Arquitecto.

\begin{abstract}
Resumen
Los barrios históricos situados en las faldas de las construcciones defensivas de las ciudades se han quedado, a menudo, excluidas de los avances urbanísticos y sociales (viviendas insalubres, escasos servicios públicos, inexistentes equipamientos, rentas bajas) sufriendo un proceso de vaciado y degradación social. Por otro lado, los castillos que coronan la mayoría de las poblaciones españolas siguen siendo el mayor foco de atracción turística, creándose asi una barrera física y social entre ambos espacios, históricamente relacionados.

Enfocado a los niños y con la intención de dar a conocer de una manera lúdica su entorno más inmediato, zonas desprotegidas o con escaso interés turísticos por la lacra social que les envuelve, se ha desarrollado un programa pedagógico cultural en torno a los bienes culturales dentro de un lugar con riesgo de exclusión social, haciendo que les pertenezca e invitando al resto de la sociedad a interesarse por ese barrio históricamente rico de vida.
\end{abstract}

Palabras clave: integración, participación ciudadana, barrio, monumento, educación, patrimonio, inclusivo.

\begin{abstract}
The historic neighborhoods located on the slopes of the defensive buildings of the cities have often been excluded from urban and social advances (unhealthy housing, few public services, nonexistent facilities, low incomes) undergoing a process of emptying and social degradation. On the other hand, castles that top the majority of Spanish populations continue to be the main focus of tourist attraction, thus creating a physical and social barrier between the two spaces, historically related.

Focused on children and with the intention of making public in a playful way their immediate environment, and the unprotected or lacked of interest areas, we have developed a cultural and pedagogical program developed around cultural assets within from a place at risk of social exclusion, making it belong to them and inviting the rest of society to be interested in that historically rich neighbourhood of life.
\end{abstract}

Keywords: integration, citizen participation, neighbourhood, monument, education, heritage, inclusive. 


\section{Introducción}

La primera mitad del siglo XXI se está caracterizando en el ámbito profesional de la arquitectura como un momento de renovación. En el campo del patrimonio arquitectónico las oportunidades son muchas. En ocasiones, los equipos pluridisciplinares que intervenían directamente sobre el bien patrimonial han pasado a formar parte activa en los programas de sensibilización de la población en pro de "adoptar" su patrimonio.

Tras más de 15 años trabajando en intervención sobre el patrimonio construido, nuestro equipo ha podido constatar en numerosas ocasiones en el entorno de su propia obra el desapego de la ciudadanía para con su patrimonio. A menudo nos hemos encontrado con la mirada de curiosos vecinos que preguntaban a las puertas de la ermita, molino, pajar o lavadero... "pero ¿van a gastar dinero ahí? si eso no tiene ningún valor...".

Como gestores de ese patrimonio, las administraciones locales (y no tan locales) a menudo apuestan de manera muy centralizada por la protección y recuperación física de los elementos patrimoniales, descuidando la realización de un proceso previo o simultáneo al proyecto de intervención arquitectónica, que sería el de gestión y difusión, en el que intervengan todos los agentes implicados en el bien.

No es tarea sencilla involucrar de una manera activa al colectivo civil (Consejo de Europa, 2005) ya que se trata de un colectivo no siempre motivado, no necesariamente profesional y a menudo desconocedor. Es necesario facilitar herramientas para que surja el interés en la puesta en valor del patrimonio y su salvaguarda y eso pasa, necesariamente, por la educación patrimonial. Y es que no se puede valorar aquello que no se conoce.

Este escenario posibilitó que nuestro equipo de didáctica del patrimonio desarrollara, en el año 2019, el proyecto HA del castillo!! (Historia y Acción social en torno a las fortalezas españolas).

\section{2. Ámbito de actuación}

El proyecto se desarrolla en barrios ubicados a las faldas de las históricas fortificaciones que coronan las poblaciones. Dichos distritos, por su orografía y urbanismo heredado, cuentan de manera sistemática con una serie de problemáticas de complicada solución.

El Ministerio de Sanidad, Servicios Sociales e Igualdad clasificaba en 2011 los cascos históricos como una de las grandes "familias" del Patrimonio Histórico-Artístico y resaltaba que se conforman, mayoritariamente, a partir de arquitectura popular, la cual se caracteriza por ser vulnerable, dada la dificultad intrínseca y cuantitativa de valoración que posee y la escasa protección que se le suele asignar (Juncá, 2011).

El mismo documento ahonda en una descripción tipológica más detallada donde se hace referencia a las Ciudades medievales concéntricas y de crecimiento orgánico asentadas en bordes de ladera o colina clasificadas como ciudadesfortaleza.

Esos barrios se caracterizan por ser de difícil acceso debido a barreras arquitectónicas insalvables, como las pendientes que presentan sus estrechas calles. Otro de los problemas que impiden el crecimiento y que alejan estas zonas del resto de la ciudad es la escasez de transporte público y dificultad de acceso hasta la propia vivienda con transporte privado. Así como el transporte encuentra problemas de acceso, los servicios de saneamiento y recogida de basura también. Son barrios que carecen de infraestructuras de saneamiento. Otro tipo de suministros se encuentran en muchos casos mermados derivando todo ello en viviendas no adecuadas a la normativa de habitabilidad y salubridad. En términos de datos sociales y educativos presentan una de las tasas de renta bajas, así como elevado abandono del sistema educativo por parte de los menores de 16 años.

Todas estas características evidencian que los barrios que nos ocupan son propicios a sufrir la problemática de la exclusión social. Se trata de condicionantes que empujan a un abandono sistemático de la zona a los pies de la fortaleza, que en muchas ocasiones puede llegar a convertirse en un gueto, lo cual potencia una fractura de las comunicaciones entre la población del ensanche (nuevos barrios bajo ladera) y su foco de interés turístico. La solución adoptada en la 
mayoría de los casos estudiados es realizar una buena infraestructura de comunicación directa hasta la fortaleza (vía rodada) y un espacio de aparcamiento para asegurarse la llegada del turismo a las puertas del bien patrimonial.

\section{El proyecto}

Como así lo establecería la Convención de Budapest (2011) con el Patrimonio Mundial (y aquí ya lo hemos mencionado con la Convención de Faro (2005)), destacamos las cinco C (aumentar la Credibilidad como testimonio representativo y equilibrado / velar por la Conservación eficaz de los bienes / fortalecimiento de Capacidades a fin de facilitar la comprensión / Comunicar el conocimiento que el público tiene del patrimonio y / reforzar el papel de las Comunidades) que se extrajeron como orientaciones estratégicas y que nosotros recogemos como plan de acción en nuestro proyecto sobre educación patrimonial.

El proyecto propuesto pretende plantear estrategias y establecer mecanismos que permitan a la ciudadanía aproximarse a la herencia patrimonial que les rodea, aumentando la implicación de los ciudadanos cuyo rol dejaría de ser, como viene siendo habitual, el de mero espectador pasivo ante el elemento patrimonial visitado. Desarrollamos unas dinámicas y estrategias de enseñanza en torno al patrimonio cultural construido siguiendo así mismo las directrices del "Plan Nacional de Educación y Patrimonio" establecidas por el Instituto de Patrimonio Cultural Español (2015) Se conciben como una serie de acciones pedagógicas-culturales en torno al monumento en lugares en riesgo de exclusión social. A través de grandes representaciones en cartón reciclado de las fortalezas se pretende, por una parte, acercar estos vecindarios al público, investigando bajo un prisma de participación arquitectónica, sociológica y urbana, mejorando la percepción de estos vecindarios; y por otra, establecer mecanismos que permitan a los ciudadanos acercarse al patrimonio que los rodea y hacerlos más conscientes de que estos representan una parte muy importante de su historia.

Así mismo, con la voluntad de utilizar la creatividad, la arquitectura, la cultura y el patrimonio como herramientas para la inclusión social, y con el fin máximo de buscar la sensibilización de la población para con su patrimonio, se propuso el proyecto "HA del castillo!! Historia y Acción social en torno a la las fortalezas españolas", proyecto desarrollado gracias al cofinanciamiento de la Fundación BBVA a través de las becas Leonardo para investigadores y creadores culturales, en la categoría de Arquitectura e Ingenierías ${ }^{1}$.

Animados por el equipo docente especializado, la experiencia se convierte en un verdadero tiempo de enseñanza para el niño donde se combina la observación, la reflexión, la manipulación, la crítica y la expresión. Un proyecto educativo donde se cultivan las capacidades de los más jóvenes desarrollando el aprendizaje del Patrimonio en nuestras ciudades y las tradiciones culturales. Por otra parte, al tratarse de una actividad educativa, complementaria al programa educativo escolar, se fomenta el trabajo en equipo, la organización y el promover y desarrollar la habilidad manual y desarrollar un espíritu crítico en relación con su entorno, responsabilidad, ciudadanía y respeto hacia los demás.

Como complemento de estos objetivos establecidos, siempre hemos valorado ciertas premisas en el trabajo con los niños como son el aprendizaje de los valores del juego y la reutilización y reciclaje de material de uso común.

Y por supuesto, como objetivo central del trabajo pedagógico destacamos la reflexión y la sociabilización para que se entienda la importancia del Patrimonio, en concreto del patrimonio más cercano, y así llegar a cultivar la capacidad de transmisora de los jóvenes en su entorno social y familiar.

\section{Metodología}

"Para desarrollar un trabajo en la didáctica de los bienes patrimoniales es fundamental que se impliquen profesionales de diversos ámbitos además de aquellos consagrados al Patrimonio y a la Arquitectura, se debe contar con agentes especializados en la educación de nuestros hijos. Educadores, animadores culturales, artistas, diseñadores completan el equipo, compartiendo su pasión por la Arquitectura y el Patrimonio" (Laumain et al., 2014, p. 150).

\footnotetext{
${ }^{1}$ La Fundación no se responsabiliza de las opiniones, comentarios y contenidos incluidos en el proyecto y/o los resultados obtenidos del mismo, los cuales son total y absoluta responsabilidad de sus autores.
} 
La metodología aplicada en nuestro caso de estudio, las fortalezas españolas en pequeñas poblaciones, se divide en:

\subsection{Muestreo}

Una fase fundamental para realizar un estudio es obtener unos resultados fiables para lo cual se necesita la mayor cantidad de datos posibles. Por lo general resulta imposible realizarlo en la totalidad de los casos obtenidos, para lo cual se realiza un muestreo. El proyecto comenzó con una fase de documentación y toma de contacto. En un primer momento se procedió al vaciado documental sobre poblaciones en las que exista, o haya existido, alguna fortaleza, se creó una base de datos que serviría, posteriormente para establecer contacto con las poblaciones potencialmente elegibles a través de teléfono o correo electrónico.

Las principales fuentes de información fueron páginas web con información histórica o turística, publicaciones especializadas, información obtenida a través de redes sociales y información recabada en FITUR 2019, donde se presentó el proyecto y se mantuvieron reuniones con representantes de distintas poblaciones y regiones.

Paralelamente a la fase de documentación, se creó de una página web www.hadelcastillo.es creada con dos objetivos claramente definidos. Por una parte, dar a conocer el proyecto y ofrecer a las poblaciones la posibilidad de pasar a formar parte del mismo y, por otra, servir como escaparate de las actividades que se van realizando, y funcionar como archivo fotográfico y testigo digital de la evolución del proyecto.

\subsection{Selección}

Tras la fase de muestreo, se realizó una primera selección de poblaciones que según el criterio establecido por el equipo, se ajustaban al perfil solicitado. Se contactó con responsables culturales, concejales, agentes de desarrollo local... ampliándoles la información del proyecto y solicitándoles la cumplimentación de un cuestionario online, cuyos resultados servirían como instrumento de selección posterior.

El empleo de cuestionarios o encuestas como herramientas de investigación es una técnica ampliamente utilizada que permite obtener datos de modo rápido y eficaz. Con el objetivo de aproximarnos a la realidad de cada población interesada, se diseñaron una serie de preguntas de respuesta cerrada que nos permitiesen conocer las particularidades de la población. Más allá de los datos generales, nos centramos en la relación física de la población con su castillo o fortaleza y el estado en el que se encontraba el casco antiguo (Fig. 1). Ambas respuestas resultaban claves para determinar qué poblaciones se ajustaban al perfil del proyecto.
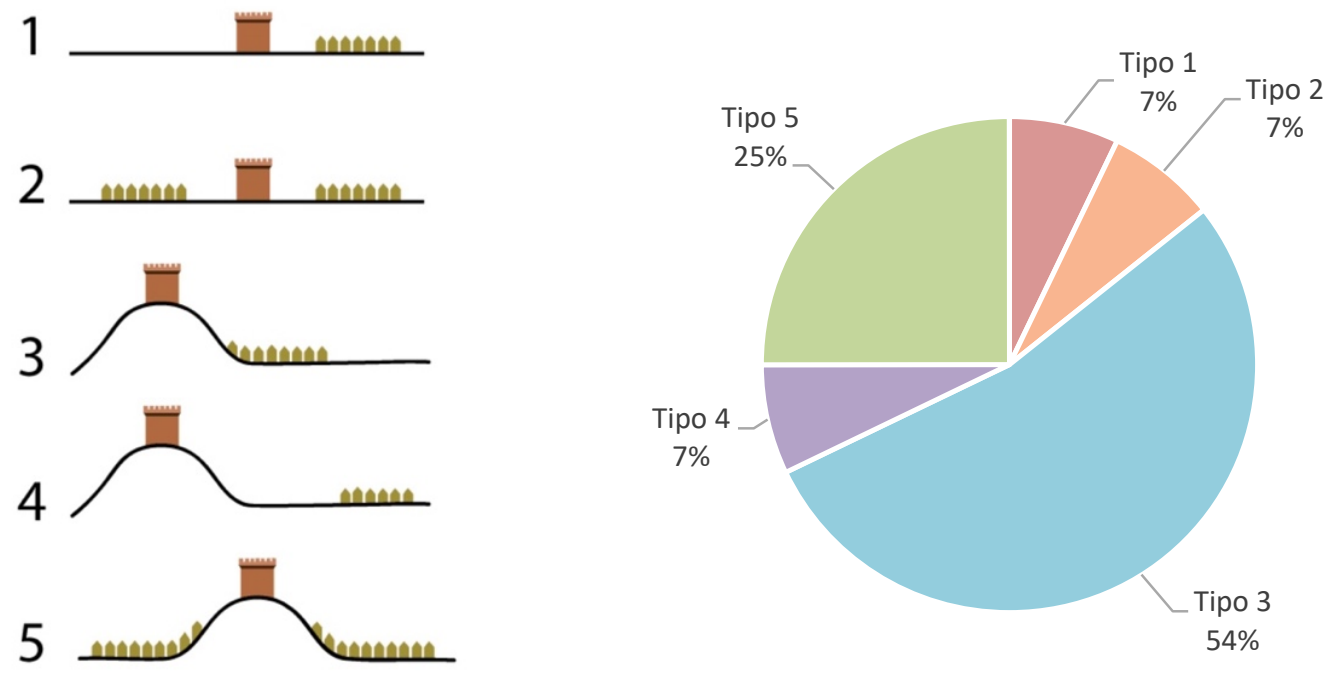

Fig. 1 Relación orográfica población/castillo 


\subsection{Análisis y elaboración de propuesta individualizada}

Una vez seleccionada una población (Fig. 2) comenzaba el trabajo específico consistente en la investigación sobre el bien patrimonial y el diseño, elaboración de planos y realización de maquetas que permitieran la construcción en cartón reciclado de la reproducción del castillo o fortaleza. Además se realizaban análisis urbanísticos de la población, conjuntamente con los agentes locales, para seleccionar la ubicación de las actividades a desarrollar in situ.

Tras un segundo formulario con datos más específicos de la población seleccionada, donde se establecían las zonas más degradadas del barrio, las necesidades reales de implementación de servicios, tasas de escolarización, rentas del vecindario...) se definen los valores y elementos patrimoniales potenciales a resaltar del centro histórico, por sus valores arquitectónicos, sociales, históricos... que servirán de apoyo a las actividades a realizar posteriormente.

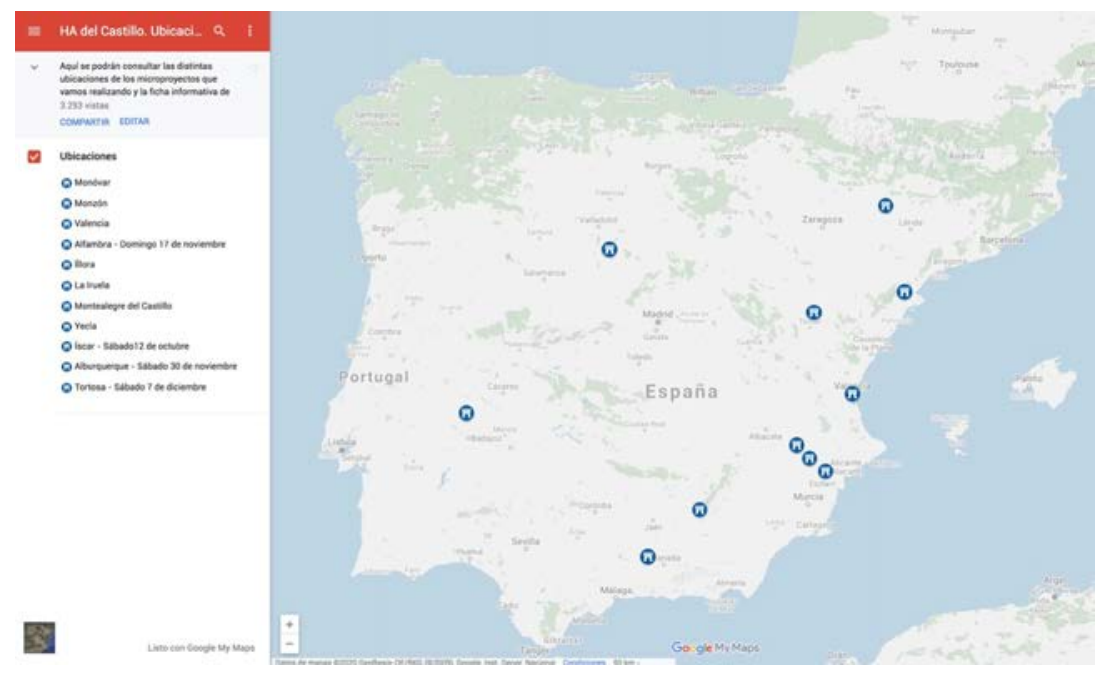

Fig. 2 Municipios seleccionados para el proyecto

\subsection{Primera sesión in situ}

A raíz de las primeras tomas de contacto se realiza una primera actividad didáctica que tiene lugar fuera de los límites del centro histórico, con niños de la zona de ensanche de la misma población. Esa actividad tiene doble objetivo:

Por una parte, hacer un llamamiento directo a los habitantes "ajenos" al espacio histórico de las inmediaciones del castillo, ya que se ha comprobado que en estos casos, la gran mayoría de la población nunca ha visitado esa parte de su municipio, bien por desinterés, bien por pudor ante la infamada inseguridad de sus calles, o bien por marcar el límite de segregación por culturas desiguales;

Por otra parte se pretende hacer a los vecinos conocedores de los valores patrimoniales de su ciudad. De nuevo la experiencia nos muestra, desgraciadamente, que gran parte desconocen la historia, los monumentos o los espacios históricos y relevantes de su ciudad, ende de ese barrio.

Como ocurrieran en la población de Monzón (Huesca), se pudo comprobar que un 74\% de los participantes (63 niños participaron en esta primera jornada) nunca habían transitado la zona del casco histórico donde se iba a realizar la segunda actividad, y lo mismo ocurría con sus familiares que les acompañaban. Se pudo concluir que efectivamente existe un gueto con esos centros históricos que no poseen una explotación turística/cultural que se desarrolla en paralelo o acorde a lo que ocurre con su monumento defensivo, hito en la mayoría de los casos del patrimonio cultural del municipio.

Con esa realidad queríamos beneficiarnos del hecho de que la población joven posee una potente capacidad transmisora en su entorno familiar, y por tanto lo entendemos en este proyecto como el foco activo que logra el objetivo impuesto por el equipo de trabajo. Por ello, esta primera acción sirve de llamamiento a participar en la actividad lúdica que tendrá lugar posteriormente a las faldas del castillo. 
Tras esa primera experiencia con los más jóvenes se hace una puesta en común de impresiones y conclusiones, donde se valora el grado de aceptación del juego a través de la respuesta de los participantes a repetir posteriormente la experiencia, esta vez en un barrio casi prohibido para ellos.

\subsection{Realización del taller final}

Se desarrolla una actividad lúdica como elemento propiciador de la interacción y cohesión social a las faldas del castillo. En un espacio abierto y amplio, siempre en el núcleo del barrio desprotegido, poco conocido para el resto de la población pero un espacio de juego común para la población infantil o juvenil del barrio.

Se aplica la propuesta didáctica desarrollada por nuestro equipo donde se construye a una escala atractiva para los niños, la fortaleza que corona su ciudad. Esa maqueta, representando la realidad actual o bien reinterpretando las ruinas en las que gran parte de nuestros castillos se encuentran, sirve de contenedor de los juegos, todos ellos en torno a los valores patrimoniales del lugar anteriormente analizados.

Supone la transformación del hito territorial en instrumento real del aprendizaje y, por lo tanto, del conocimiento a través del juego. El modelo que se propone dispone de un elemento clave: centrar la línea de acción didáctica en el juego en grupo.

Por otra parte, se trabaja la importancia de la heráldica y el reconocimiento de sus símbolos como comunidad, así como la identificación por cada uno de los participantes de la ubicación del castillo en un sencillo plano de la población y la colocación de símbolos identificando castillo/domicilio, escuela/ayuntamiento, ocio/comercio, etc.

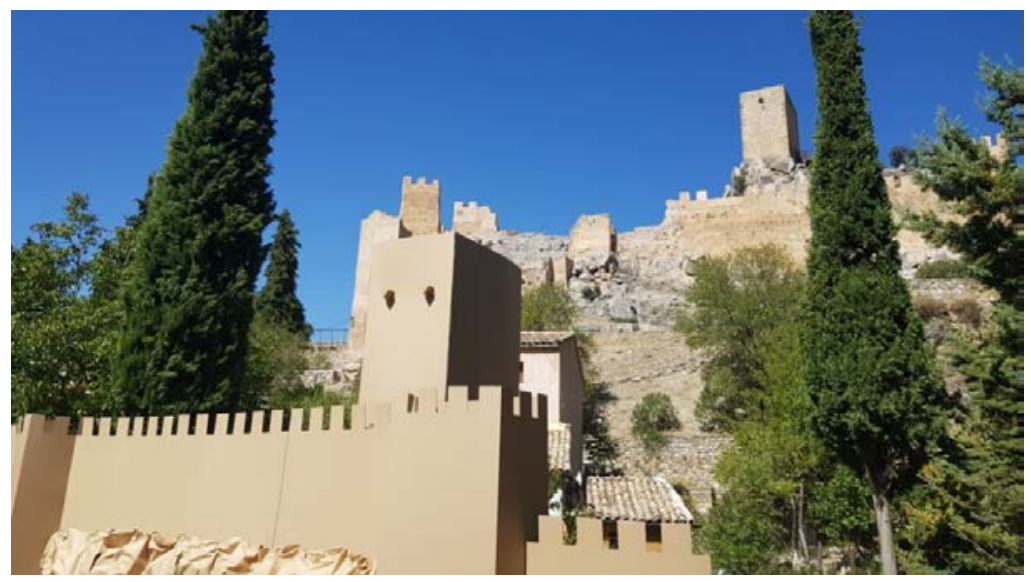

Fig. 3 Reproducción (primer plano) y castillo de La Iruela (segundo plano) en Jaén

\section{Alcance del proyecto}

Una de las premisas de este proyecto consistía en extender a lo largo de la geografía española las actividades de didáctica patrimonial que venimos desarrollando habitualmente en el ámbito de la Comunidad Valenciana. En efecto, las primeras poblaciones interesadas en formar parte del proyecto fueron aquellas más cercanas, y resultó de mayor complejidad llegar a poblaciones que nunca habían oído hablar de nuestro trabajo.

El recorrido por España comenzó en la localidad alicantina de Monovar (Alicante) cuya fortaleza en la actualidad se reduce a una torre del homenaje truncada. Tras un parón de varios meses, nos trasladamos a la localidad oscense de Monzón, en la que recreamos la fortaleza de origen medieval que corona su centro urbano. Andalucía acogió dos jornadas del proyecto, una en la provincia de Granada, en Íllora, y otra en La Iruela (Jaén) (Fig. 3). En la provincia de Valladolid disfrutamos confeccionando las atractivas torres y almenas circulares, y luchando contra el viento... ya que tuvimos que enfrentarnos a condiciones meteorológicas adversas en el municipio de Íllora. Del mismo modo ocurriría semanas después en la localidad de Alfambra (Teruel), donde ante el riesgo de nevadas, se tuvieron que trasladar las actividades al polideportivo municipal. Allí pudimos comprobar que ante la desaparición de la mayoría de las construcciones originales del castillo (torres, caballerizas, aljibes, viviendas...) la percepción para los alfambreños de su 
castillo se reduce al Cristo Redentor o la zigzagueante subida al castillo (tal y como se puede ver en los dibujos que hacen los niños a lo largo de la actividad) (Fig. 4). La siguiente parada fue Yecla (Murcia) donde a pesar de que la actividad estaba programada para unos 40 niños, llamó tanto la atención que se acercaron más de 120, teniendo que improvisar juegos y material complementario y poniendo a prueba a nuestro equipo de 3 educadores patrimoniales.

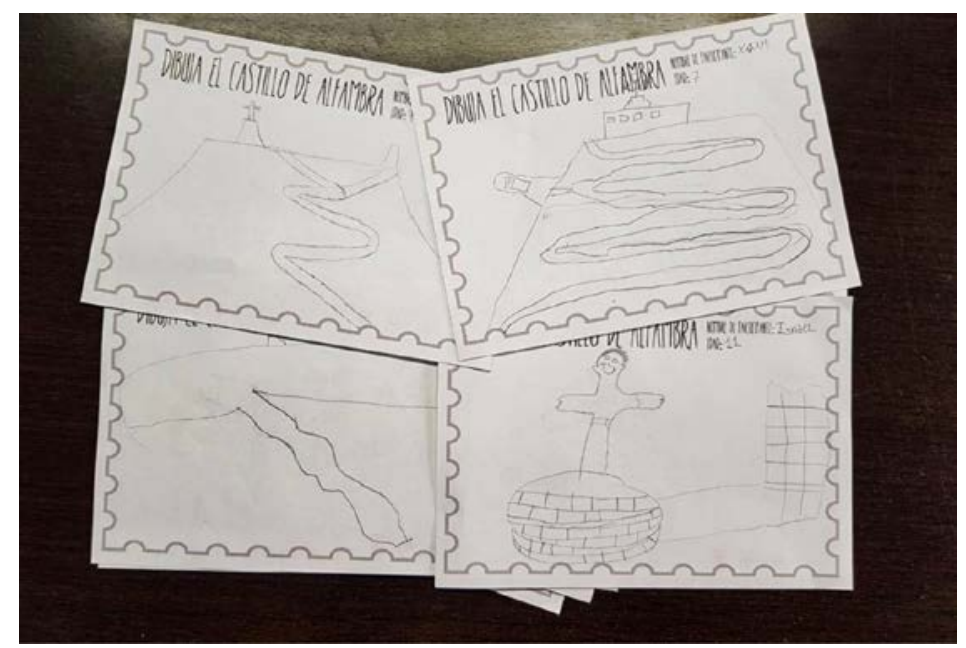

Fig. 4 Ilustraciones de los participantes en Alfambra (Teruel), reproduciendo su castillo

Continuamos en Montealegre del Castillo (Albacete), donde la actividad sacó a la luz, mediante el dibujo de los niños, que se ven más las antenas de telefonía que el propio castillo. En el mes de diciembre, para concluir, se trabajó en Albuquerque (Badajoz) y por último en Tortosa (Tarragona), donde algún participante nos remarcó "antes era un castillo, pero ahora es un hotel y no me gusta así" (Fig. 5).

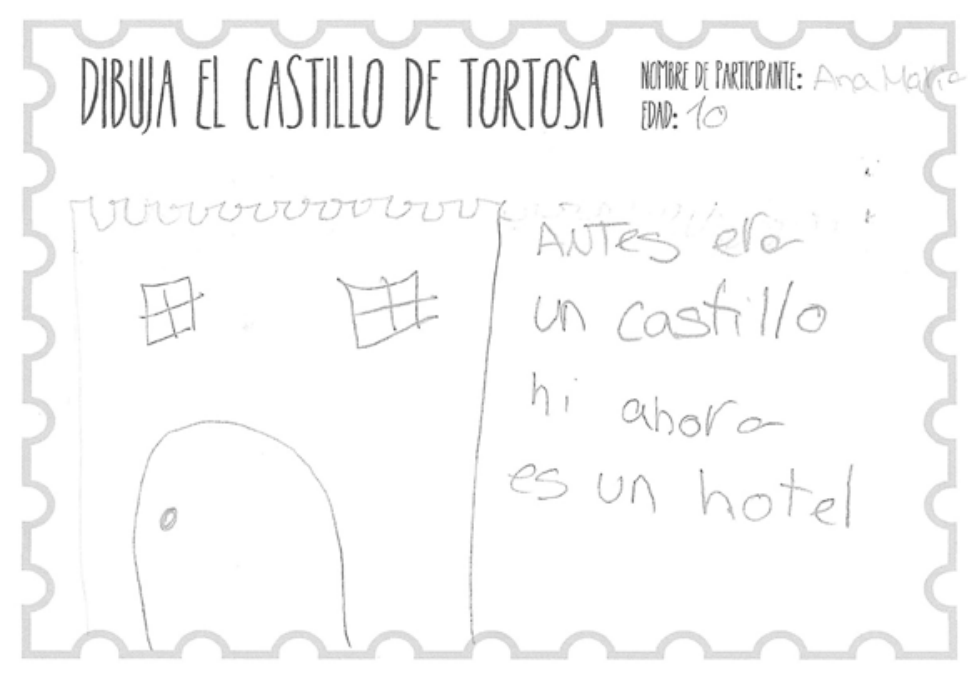

Fig. 5 Ilustración de una participante donde expresa la realidad del castillo de Tortosa (Tarragona)

Cabe remarcar que las jornadas educativas del proyecto HA del castillo!! han sido seleccionadas para formar parte de las actividades de las Jornadas Europeas del Patrimonio (Fig. 6), promovidas por el Consejo de Europa.

\section{Resultados y conclusiones}

Decía el sociólogo Francesco Tonucci (1996) que hay que trabajar para que la ciudad sea más apta para los niños, y entonces será apta para los ancianos, minusválidos y los adultos en general. 
La ciudad, concretamente los barrios, son lugares donde socializamos, nos relacionamos, y donde tenemos contacto con los demás más allá de nuestro habitual espacio de confort, nuestra casa, y no siempre resulta un espacio cómodo para el juego y para sus aventuras.

De las experiencias vividas y los datos obtenidos a lo largo de los 18 meses de trabajo se ha podido constatar que el bien patrimonial es una efectiva herramienta para la cohesión social y su utilidad pasa por saber posicionarlo dentro del barrio en el que se enclava a través de la memoria e identidad colectiva de los vecinos.

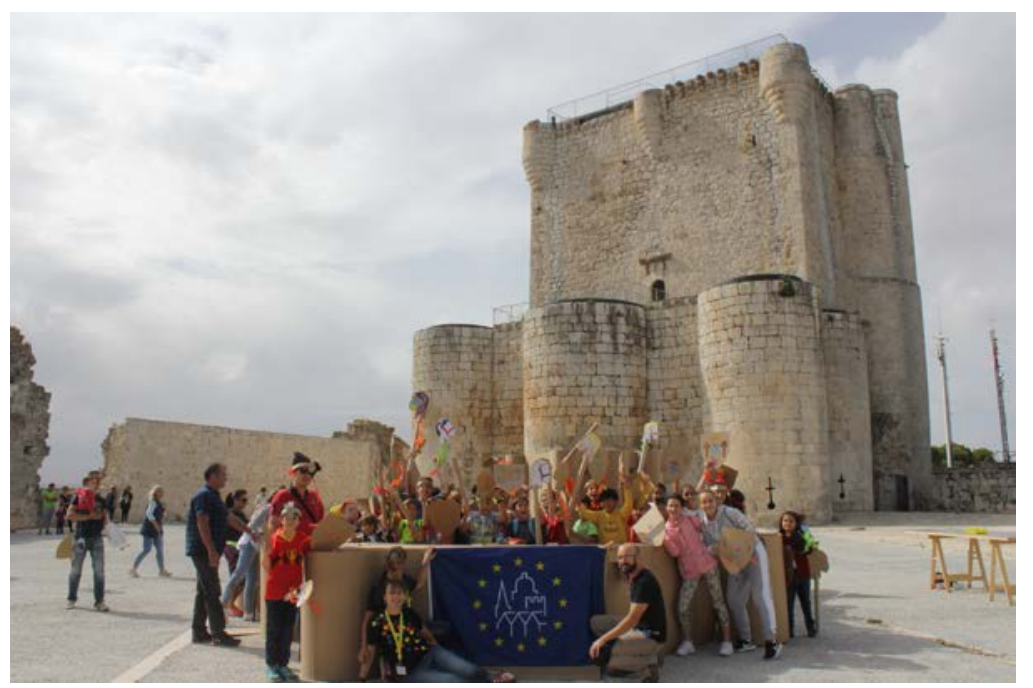

Fig. 6 Grupo de trabajo en Íscar (Valladolid), actividad incluida en las Jornadas Europeas del Patrimonio

Los dibujos realizados por los niños y las conversaciones con padres y madres en cada una de las sesiones nos han ayudado a detectar que existe una marcada diferencia en la relación ciudadanía-patrimonio entre las poblaciones en las que anteriormente se han realizado actuaciones de gestión y puesta en valor el bien patrimonial frente a aquellas en las que no se ha actuado o se han realizado únicamente acciones de conservación o restauración que quedan alejadas de la percepción del ciudadano de a pie. Se hace necesario trabajar en ese sentido, haciendo partícipe a la ciudadanía de los valores inherentes al bien patrimoniale e implicándola de modo activo en la puesta en valor del mismo. No podemos olvidar que solo se protege y conserva aquello que se conoce y se valora.

El uso didáctico de los bienes patrimoniales debe conseguir emocionar a sus receptores, que deben disfrutar descubriendo su historia, su patrimonio, y generando las diferentes lecturas superpuestas como único modo de alcanzar la accesibilidad total del mismo.

\section{Referencias}

Consejo de Europa. (2005). Convenio marco del consejo de europa sobre el valor del patrimonio cultural para la sociedad. Recuperado de https://rm.coe.int/16806a18d3

Juncà, J. A. (2011). Accesibilidad Universal al Patrimonio Cultural. Fundamentos, criterios y pautas. Madrid: Real Patronato sobre Discapacidad.

Laumain, X., López, A., y García, V. (2014). Patrimonio para niños: una propuesta didáctica para la sensibilización hacia el Patrimonio Cultural. En O. Fontal y A. Ibáñez (Coords.), Reflexionar desde las experiencias. Actas del II congreso internacional de educación patrimonial (pp. 147-158). Madrid: IPCE/OEPE.

Ministerio de Educación, Cultura y Deporte. (2015). Plan Nacional de Educación y Patrimonio. Recuperado de $\underline{\text { https://sede.educacion.gob.es/publiventa/descarga.action?f_codigo_agc }=15106 \mathrm{C}}$

Tonucci, F. (1996). La ciudad de los niños. Madrid: Fundación Germán Sánchez Ruipérez. 